Research Article

\title{
Muscle Injury in Bodybuilding Based on Mesoporous Multifunctional Nanomaterials for Sports Rehabilitation Training
}

\author{
Changjun Zhao \\ Department of Physical Education, Guangxi University of Chinese Medicine, Nanning 530200, Guangxi, China \\ Correspondence should be addressed to Changjun Zhao; zhaochangjun78@163.com
}

Received 10 August 2020; Revised 18 September 2020; Accepted 21 September 2020; Published 23 October 2020

Academic Editor: Tifeng Jiao

Copyright (c) 2020 Changjun Zhao. This is an open access article distributed under the Creative Commons Attribution License, which permits unrestricted use, distribution, and reproduction in any medium, provided the original work is properly cited.

\begin{abstract}
As an important means of treating diseases, chemical-based drugs have always guaranteed people's health and longevity. However, due to the nature of some drugs, their use in the medical field is limited. This study mainly discusses the treatment of muscle damage based on sports rehabilitation training mesoporous multifunctional nanomaterials in bodybuilding. The biological characteristics of the targeted control of mesoporous multifunctional nanomaterials for target drugs were studied by analyzing the regeneration of skeletal muscles of mice after gastrocnemius strain under computer control. The electrostatic interactions modified by the consensus binding between AS1411 and Dimer-PPTcDA on the surface of mesoporous silicon are used to block the pores, and the release of objective molecules depends on the concentration of the drug in the tendon cells and the time of action. In this system, AS1411 has both the characteristics of the target substance and the active site of the targeted stimulus response in the cell. After FAM is labeled AS1411, the fluorescence of FAM can be used to monitor the release of the drug in real time, so as to directly release the drug to the lesion, maintain local effectiveness, and greatly improve the biological activity of the drug. In addition, in the safety analysis of mesoporous multifunctional nanomaterials on cells, if the concentration of nanoparticles is $90 \mu \mathrm{g} / \mathrm{mL}$, the cell survival rate is almost $100 \%$. The results show that the mesoporous multifunctional nanomaterials have low cytotoxicity and cell activity is not affected. The smaller the particle size of mesoporous silicon, the easier it is to invade the focus cells. In addition, confocal microscopy imaging has also effectively demonstrated the targeting effect of mesoporous multifunctional nanomaterials on cells.
\end{abstract}

\section{Introduction}

In recent years, great progress has been made in the application of nanotechnology and its biomedical or chemical sensing fields. Due to the special advantages of inorganic nanoparticles, many functional nanomaterials have gradually expanded from traditional organic-based nanomaterials to hybrid nanocomposite materials.

As a porous material, the mesoporous multifunctional nanomaterials have extremely high loading efficiency for drugs due to their large and uniform pore size, and the mesoporous multifunctional nanomaterials have wide applications in many fields. The nature of its catalytic drugs will make it highly valuable in medicine.

Molecular imaging is not only widely used as an important detection technology for cancer diagnosis in the field of medical imaging, but also as a treatment method for cancer in recent years. Yanyan believes that multifunctional carbon-based nanomaterials (MCBNs) with unparalleled optical, electronic, and thermal properties have attracted more and more interest and show the greatest hope in the field of biomolecular imaging and therapy. Therefore, carbon-based nanomaterials have a large number of potential applications in the field of biomedicine, such as bioimaging, drug delivery, and tumor treatment [1]. His research only stayed at the theoretical level and did not use experimental exploration. Sustainable and cost-effective energy production has become the key to meeting current energy needs. Paul believes that a cheap, scalable, efficient, and reliable catalyst must be developed. Carbon-based heteroatomdoped 3D and mesoporous electrodes are very promising as catalysts for electrochemical energy conversion and storage. 
Various carbon allotropes doped with various heteroatoms can be used to mass-produce electrode materials cost-effectively. 3D porous carbon electrodes have many advantages, such as 3D conductive paths for efficient electron transport and porous channels that promote electrolyte diffusion. However, synthesizing and functionalizing isotropic 3D carbon structures are challenging. He summarized various synthetic processes of $3 \mathrm{D}$ porous carbon materials to understand their physical and chemical properties [2]. His research did not give a specific process for storing energy by doping carbon-based heteroatoms. Carbon-based nanomaterials have been developed for photothermal cancer treatment, but it is still a huge challenge to manufacture their multifunctional counterparts with simple methods, good biocompatibility and dispersion, and efficient cancer therapeutic methods. Li et al. have developed an alternative multifunctional nanoplatform based on carbon silica nanocapsules, which have gold nanoparticles (Au @ CSN) in the cavity for cancer treatment. The encapsulated chemical doxorubicin can be released from Au @ CSN with mesoporous and hollow structure in the manner of near-infrared light and $\mathrm{pH}$ stimulation, which is beneficial to spatiotemporal treatment to reduce off-target toxicity. Nanocapsules with efficient photothermal conversion and excellent biocompatibility have achieved synergy between photothermal and chemotherapy [3]. Exercise under hypoxic conditions represents additional stress associated with normoxia. Santos believes that hypoxia can induce inflammation of oxidative stress, which may increase after exercise. In addition, vitamin E supplementation may reduce oxidative stress and inflammation caused by hypoxia during exercise. He evaluated the effect of vitamin E supplementation $(250 \mathrm{mg})$ on inflammatory parameters and cell damage after simulated hypoxia exercise at an altitude of $4200 \mathrm{~m}$. Nine volunteers were supplemented with vitamin E 1 hour before exercise and performed three 60-minute exercises (maximum oxygen uptake $70 \%$ ) under normoxia, hypoxia, and hypoxia for a week. Blood was collected before exercise, after exercise, and 1 hour after exercise to measure inflammation parameters and cell damage [4]. His research only judged the effect of oxygen content on body health and did not analyze the exercise time in detail.

In this study, the targeted characteristics of mesoporous multifunctional nanomaterials were used to treat the injured parts in the bodybuilding process in a timely manner, which improved the activity of the drugs and quickly recovered the injured parts.

\section{Mesoporous Multifunctional Nanomaterials}

\subsection{Advantages of Mesoporous Multifunctional} Nanomaterials. The particle size of mesoporous multifunctional nanomaterials and ribose proteins, natural carriers, etc., and the structure of the hydrophilic polymer shell of ordinary long-circulating nanoparticles are difficult to be rejected by cells [5]. The PEG surface of the mesoporous multifunctional nanomaterials and the hydrophilic chain of PEO make it have good flexibility and can pass through the space with sufficient plasma density. Its conformation is formed to prevent the aggregation of particles. Therefore, stable mesoporous multifunctional nanomaterials will spend a lot of time on cycling. The CMC of polymers is usually low (approximately 5-10 MOL), so the entire nanosystem has very high thermodynamic stability. The activity of mesoporous multifunctional nanomaterials is shown as follows:

$$
V \%=\frac{[A]_{\text {sample }}-[A]_{\text {blank }}}{[A]_{\text {positive control }}-[A]_{\text {blank }}} \times 100 \% .
$$

where $[A]_{\text {sample }}$ is the sample quality, $[A]_{\text {blank }}$ is the quality of the blank control, and $[A]_{\text {positive control }}$ is the corresponding positive control. In addition, mesoporous multifunctional nanomaterials are mainly used for intravenous injection. The thermodynamic (depolymerization potential energy) and kinetic (depolymerization rate) stability of polymer micelles after intravenous injection and vascular dilution must also be considered. The mesoporous multifunctional nanomaterials must be stable enough to avoid sudden drug release and must exist in the form of nanoparticles for a relatively long time to ensure that their concentration in the target area is sufficiently high [6]. The critical mesoporous multifunctional nanomaterial concentration indicates that when the monomer concentration is lower than this value, the nanoparticles begin to decompress, but due to the dynamic stability, the decompression speed of the nanomaterial particles is determined. However, many binary block copolymerized nanoparticles have excellent kinetic stability and will slowly dissociate the monomer after largescale dilution. Therefore, the polymer nanoparticles will be diluted below the monomer CMC after intravenous injection, but due to their kinetic stability, they are suitable for drug delivery. This is also the biggest difference between high-resolution mesoporous multifunctional nanomaterials and ordinary small-molecule surfactants $[7,8]$.

2.2. Nanodrug Carrier. For the present, mesoporous multifunctional nanomaterials have many advantages, such as proper improvement of drug dynamics and biological distribution, improvement of biodiversity, and reduction of side effects. However, the method of further achieving the localization and release of drugs at the injured site is still a compelling research topic. Localized medications have high specificity for the injured site, which can reduce the dosage to a certain extent, but will be limited by the injury. Systemic administration is still the preferred method of administration for most types of muscle damage. Therefore, the discovery of drug or gene release strategies at the site of muscle injury is the focus of nanomaterial development. Compared with previous drug preparations, nanodrug carriers have many advantages [9].

(1) Optimized drug delivery path: nanodrug carrier strengthens the drug delivery mode, multilayer carrier structure, and surface hydrophilic layer, to avoid the immune elimination of nanodrug carrier after entering the body, achieve long-term drug circulation, and shorten the drug delivery time. 
(2) Controlled release: the advantage of the size of the nanomaterials carrying the drug is that it can slowly release the drug at the targeted site through the intercellular space and through the blood-brain gate. In terms of corresponding drug treatment, targeted ligands are transplanted to the surface and bind to receptors in different tissues to achieve targeted control and drug release [10].

(3) Reduce toxicity: encapsulating drugs in a closed environment through nanodrug carriers can improve the stability of the drugs to the external environment, prevent the drugs from decomposing before reaching the lesion, and reduce the systemic drug concentration. Controlled release can also maintain a stable drug concentration in the blood and reduce drug toxicity and side effects [11].

(4) Improving absorption: nanodrug carriers are mostly carried to cells through the terminal nervous system, which can improve the transparency of the cell membrane and the compatibility of the compound during transportation, increase the absorption rate of the drug, and make the absorption efficiency of the drug larger. At this time, statistics on drug absorption and drug loading are required [12]:

$$
\begin{aligned}
& \mathrm{EE} \%=\frac{C_{\text {sample }} \times V}{M_{\text {drug }}} \times 100 \%+C_{\text {sample }} \times V_{\text {min }}, \\
& \mathrm{DL} \%=\frac{C_{\text {sample }} \times V}{\left(C_{\text {sample }} \times V+M_{\text {micelle }}\right)} \times 100 \% .
\end{aligned}
$$

where $C_{\text {sample }}$ is the DOX concentration measured with drug-loaded nanoparticles, the unit is $\mathrm{UG} / \mathrm{mL}$, and $\mathrm{EE} \%$ represents the osmotic pressure value of nanoparticles. After analysis, the volume of a certain volume is $V . M_{\text {micelle }}$ is the total dosage and the unit is UG. DL is the component, and the corresponding unit is $\mathrm{g}$.

Ultraviolet spectrophotometry and high-speed liquid tomography are the two most commonly used methods for drug analysis in vitro. The biggest advantage of high-speed liquid tomography is that it can separate and detect multicomponent samples online $[13,14]$. However, because the components need to be separated before detection during the detection process, the analysis time of HPLC is long, the device requirements are high, and the analysis cost is high, avoiding environmental problems caused by the use of mobile phases. The advantages of UV spectrophotometry include high-speed analysis and high sensitivity. However, in multicomponent sample analysis, other components may hinder the measurement of existing components, so there are restrictions. The absorbance of each sample is measured using an ultraviolet-visible spectrophotometer. The release curve of DOX is the vertical axis with the release rate and the horizontal axis with the time. The calculation formula of the corresponding release rate $\mathrm{Er}$ is as follows:

$$
\mathrm{Er}=\frac{V_{\varepsilon} \sum_{I}^{i-1} C_{i-1}+V_{0} C_{i}}{m_{\text {drug }}}, \quad i \geq 1 .
$$

where $V_{\varepsilon}$ is the amount of replacement of the buffer storage solution, $V_{0}$ is the volume of the solution in the remote sink tube and the corresponding drug concentration in the solution released during the replacement sampling is $C_{i}(\mathrm{mg} / \mathrm{mL})$; the amount of drug in the micelle that delivers the drug for release is $m_{\text {drug }} \mathrm{mg}$.

\subsection{Modification of Mesoporous Multifunctional Nanomaterials}

2.3.1. Plasticity Characteristics. After plasticization, the tensile strength and bending elastic modulus of nanomaterials will increase. In recent years, plasticizers used include polyethylene glycerin, alanine ester, and glucose monosaccharide. These plasticizers have good biocompatibility. People perform experimental evaluation of physical properties and improve thermal analysis of nanomaterials, such as studying the specific functions of various plasticizers such as compression elasticity, elastic modulus, and glass transition temperature. According to many investigations, mesoporous multifunctional nanomaterials are relatively general plasticizers. Adding 5\% of polyethylene glycerol to nanomaterials will greatly improve the physical properties of the material, and the glass transition temperature will drop from fifty degrees to ten degrees [15].

2.3.2. Denaturation of Copolymer. With the development of polylactic acid, the demand for higher compressive strength and hydrophilicity of polylactic acid has been studied; clinical medicine needs multiple lactic acid carriers to meet the needs of drug controlled release systems to control the decomposition rate of various drugs. Polylactic acid is a part of monomers synthesized by recondensation or ringopening polymerization in a specific ratio, the characteristics of mesoporous multifunctional nanomaterials will be improved, and some new monomers can be obtained. Due to the fusion of poly (lactic acid) molecular structure, usually polyethylene (lactic acid) is copolymerized with mesoporous multifunctional nanomaterials [16].

\subsection{Effect of Mesoporous Multifunctional Nanomaterials on} Drug Release. Mesoporous multifunctional nanomaterials can also release drugs when the structure changes. Structural changes are the most important properties of nanogels. The stimulus response of nanogels will affect their structural changes with temperature changes, causing their particle size to change almost. When the mesoporous multifunctional nanomaterials are stimulated and swelled in the environment, the amount of swollen drug will be released from the gel, or the nanogel will be stimulated and contracted in the environment. As the nanogel shrinks, the loaded drug 
molecules will accumulate and dissociate. The molecules will accumulate and dissociate. Different parts of the body have different $\mathrm{pH}$ values. The normal physiological environment of the human body is about 7.5, the $\mathrm{pH}$ of the tumor tissue is about 6 , and the internal terminal system of the tumor cell has three to four different $\mathrm{pH}$ adaptation values. Therefore, the nanogel can be used as a control for tumor treatment according to the sensitivity of reduction to $\mathrm{pH}$ use at release point. As a polymer of core- $\mathrm{N}, \mathrm{N}$-lysine, and $\mathrm{N}$-isopropanol acrylamide, the loaded DOX mesoporous multifunctional nanomaterial (NLSC-NG) created the acidity of the $\mathrm{pH}$ value of the nanogel of the reactive shell surroundings. According to the corresponding structure, if the drug kills the cells, the part of the structure that does not expand will retain the contraction function and can improve the transparency of infected cells and solid tumors. Due to the huge difference in GSH content between tumor cells and normal cells, reduced mesoporous multifunctional nanomaterials can also be prepared by adjusting the receptive controlled release point of drugs $[17,18]$.

2.5. Skeletal Muscle Injury. Muscle injuries mostly occur in daily sports and sports, especially in professional athletes engaged in bodybuilding. Not only muscle injuries but also the incidence of disease is very high, the initial lack of function and structure, damage, muscle atrophy, restraint shrinking, pain, followed. Training and competition will also be affected. Now, the diagnosis of muscle damage mainly depends on the clinical symptoms, so the image examination is usually only a rough guess at the specific injury site and the injury site. The scope of ultrasound is widely used in the diagnosis of muscle injury, but the recovery and prognosis evaluation have not been widely used afterwards. The contrast ultrasound used for muscle injury is very few, but there is a small amount of muscle ultrasound repair process. However, there is a small amount of application of contrast ultrasound evaluation in the repair process of muscle damage [19].

Physiological skeletal muscle injuries can be divided into trimming injuries (fractures of tendon fibers and muscleassociated tissues) and muscle fiber injuries. In daily life, muscle pressure, muscle mass, nutritional status, training style, and balance of muscle strength all affect the occurrence of muscle damage. After the skeletal muscle injury and repair process begins, it can be divided into the following two stages $[20,21]$ :

(1) Injury stage: muscle injury due to various reasons, local hematoma formation, rupture of myofibrillar membrane, cell mass calcium overflow, increased calcium ion concentration in tissue fluid, activation of calcium-dependent protease and obstruction of mitochondrial respiratory function, and necrotic sites continuous damage. If the tissue is inflamed, macrophages will gather at the injury site and devour the diseased tissue [22].

(2) Repair stage: after muscle damage, the relevant stimulation signals are released, cell growth factors are secreted, and skeletal muscle satellite cells in the resting phase near the injury site are activated. The activated satellite cells transfer to the injury site and begin to proliferate. Some of them are the differentiation of muscle cells to form muscle tubes, which eventually merge with muscle fibers that have not been damaged before to form new lines. The proliferating satellite cells differentiate into fibroblasts, invade the extracellular space of tendon fibers, secrete extracellular matrix, form a tissue framework, and restore the connection between tendon fibers and bound tissues. At the same time, the new muscle tissue angiogenesis occurs. In this process, the damaged part is too large, and excessive proliferation of fibroblasts will produce a lot of tight coagulated tissue, hinder muscle regeneration, and form permanent muscle necrosis. Therefore, after muscle injury, in order to avoid muscle tension, it is necessary to reduce activity and affect the repair of muscle injury.

2.6. Sports Rehabilitation Training. Reasonable aerobic exercise will improve the physical condition. For patients with cardiopulmonary system, with proper diet, cardiovascular function will be normal, body fat rate will decrease, and blood fat content will decrease. Improved blood pressure and insulin sensitivity can be achieved with the goal of improving the prognosis of coronary artery disease. During exercise, the blood volume of the heart increases, the pressure of the heart increases, the blood pressure during the left ventricular dilatation increases, the subendocardial blood flow increases, the heart rate and blood pressure decrease, and the myocardial oxygen consumption decreases, thereby improving myocardial ischemia to achieve goodness purpose. On the other hand, resistance training will improve the basal metabolic rate, increase muscle mass, and reduce the proportion of body fat. After training, muscle strength and endurance will improve, and patients return to daily life, which is also helpful for work. By stretching joints and ligaments, flexible training can expand the movement area of the joints and prevent injuries to the extensive movement areas of the joints during exercise.

\section{Muscle Injury Treatment Experiment}

3.1. Experimental Reagents. The experimental reagents and names used in the experiment are shown in Table 1. The aseptic operation was strictly observed during surgery. Mice were anesthetized with cyclophospholipids (100 mg/kg). After the rats were successfully anesthetized, the parameter changes on both sides of the muscles they contacted were measured. After disinfection, $10 \%$ ethanol solution was used to collect the sterilized muscle cells for use.

3.2. Experimental Materials. The selected mice are all male mice, with a body weight ranging from $30 \pm 2 \mathrm{~g}$, and reared for 3-4 weeks. Water and food were provided for 4 weeks, and the temperature was kept at $25-30^{\circ} \mathrm{C}$ under natural light. 
TABLE 1: Experimental reagents and names used in the experiment.

\begin{tabular}{lcc}
\hline Number & Reagent name & Manufacturer \\
\hline 1 & $0.5 \%$ saline & Celestial creature \\
2 & $10 \%$ chloral hydrate & Chenxin Pharmaceutical Company \\
3 & Hematoxylin staining solution & Qingdao Yulong Company \\
4 & SuperECL Plus luminous liquid & Beijing Biotechnology Company \\
\hline
\end{tabular}

The mice were observed for a week before the experiment. The experiment was started after getting used to the environment.

3.3. Experimental Modeling. After abdominal anesthesia with $3 \%$ pentbalbiteru sodium $(0.3 \mathrm{ml} / \mathrm{kg})$, the skin was prepared for incision on both lower limbs. After disinfecting with alcohol and iodine, the mouse was laid on a sterile operating table. A longitudinal incision was made at the distal end of the gastrocnemius muscle (about $4 \mathrm{~cm}$ in length), taking care to separate the distal gastrocnemius tendon. The data connection instrument was used for parameter determination and was fixed at one end of the universal material tester. The distal tendon of the lower leg was held with vascular forceps and the tension sensor of the universal material testing machine was connected. Under the control of the computer, the gastrocnemius muscle is stretched at a speed of $4 \mathrm{~cm} / \mathrm{min}$, and the stretching load curve is recorded at the same time. When the curve ball reaches the highest point, the power was turned off immediately, so that the muscles are in a relaxed state. The vascular forceps and towel forceps were disinfected, and the fascia and skin were sterilized layer by layer after disinfecting the wound.

3.4. Experimental Grouping. 140 adult SD rats were selected and randomly divided into 7 groups. The rats are grouped as follows:

(1) Blank control group (group A, $n=20$ ): regular feeding without intervention.

(2) Immediate group (group B, $n=20$ ): a sample was taken immediately and checked according to the rat's acute gastrocnemius animal model

(3) The first week's group (group C, $n=20$ ): according to the animal model, the test was conducted after one week of feeding.

(4) Natural cure group (group D, $n=20$ ): the animal model of the acute system of the gastrocnemius of rats is caused by a normal diet without exercise intervention and natural cure. According to different sampling time, the samples are divided into 4 different control groups.

(5) Drug treatment group (group E, $n=20$ ): an animal model of acute gastrocnemius strain of mice was made, and dynamic stimulation was performed during the recovery period. According to different sampling times, they are divided into 4 groups.
(6) The medicament group using mesoporous multifunctional nanomaterials as a carrier (group $\mathrm{F}$, $n=20$ ): the animal model of the acute system of the gastrocnemius of rats is caused by the stimulation of eccentric exercise during the recovery period. According to different sampling times, they are divided into group a, group b, group c, and group $d$.

(7) Combination group (exercise method plus mesoporous multifunctional nanomaterial drug carrier) (group $\mathrm{G}, n=20$ ): induced acute gastrocnemius muscle distortion in mice, and increased central body and centrifugal stimulation during the recovery period. According to different sampling time, the samples are divided into $\mathrm{M}$ group, $\mathrm{N}$ group, $\mathrm{P}$ group, and $\mathrm{Q}$ group.

3.5. Drug Release Control. At $37^{\circ} \mathrm{C}, 120 \mathrm{~mL}$ of phosphatebuffered saline (PBS) solution was added to the beaker, and then a dialysis bag $(\mathrm{Mn}=14000)$ containing $5 \mathrm{~mL}$ of the same buffer solution and $10 \mathrm{mg}$ of drug-loaded micelles was submerged. The drug release behavior was studied under different conditions ( $\mathrm{pH}$ or reducing agent). In a prescribed time gradient, the release solution was replaced with PBS solution, and the drug release rate was measured using a drug measuring instrument.

3.6. Determination of Encapsulation Rate and Drug Loading. The encapsulation efficiency of liposomes in this study was determined by the centrifugal membrane filtration method. An appropriate amount of collected muscle cell solution was taken and centrifuged at $500 \mathrm{r} / \mathrm{min}$ for $15 \mathrm{~min}$. The resulting supernatant was filtered with a $0.22 \mu \mathrm{m}$ filter. An appropriate amount of filtrate was diluted with methanol, and the drug content in the filtrate was measured by HPLC to obtain the amount of drug encapsulated in the liposome; the original solution of muscle cells was taken to break the emulsion with quantitative methanol, and the total dose was determined by HPLC.

3.7. Determination of Drug Load. During the experiment, the centrifugal membrane filtration method was used to derive the drug encapsulation rate. Take an appropriate amount of muscle cell solution and centrifuge at $500 \mathrm{r} / \mathrm{min}$ for 5 minutes. Use $0.11 \mu \mathrm{m}$ membrane to filter the resulting clean. Dilute the stock solution containing muscle cells and record the drug content. The original muscle cell solution was emulsified with quantitative methanol, and the total dosage was determined by HPLC. 
3.8. Statistical Analysis. Prism 5.0 was used for the analysis of the original dispersing solution, Dunnett's test was used for comparison between each group and control group, and $T$-test was used for comparison between each group. This difference was considered statistically significant at $p<0.05$.

\section{Analysis of Muscle Injury Treatment}

4.1. Muscle Injury Treatment Results. Muscle healing in different groups is shown in Figure 1. Blood samples were collected from the drug treatment group, mesoporous multifunctional nanomaterials as the carrier, and the combination group at different time gradients. Serum was separated to detect $\mathrm{CK}$ concentration, and the average CK content of the three groups of mice was determined. After 24 hours of cell transplantation injury self-healing group and treatment group, serum CK levels increased significantly. After 3 days, the CK group decreased, and the cell transplantation in the combined group was reduced compared with the drug treatment group. After 5 days, the cell transplantation in both groups decreased and the CK level decreased, and the cells with muscle damage decreased further. After 7 days, the CK content of the two groups decreased steadily. The cell damage self-recovery group was always lower than the cell damage cell content of the CK treatment group, and the muscle tissue of the mice had nearnormal adrenaline kinase. The increase of CK content indicates that the muscle tissue has been damaged, but the transplantation of muscle satellite cells has greatly reduced the CK content. The damage of the muscle tissue has not continued to expand and has been effectively repaired. The content of $\mathrm{CK}$ is stable and tends to be close to the normal value. This indicates that the damage of the mouse's muscle tissue has been improved. The results of MCF-7 cell uptake indicate that intracellular drug uptake increases over time. However, the absorption of free DOX at a given time is much lower than the absorption of nanoparticles incorporating a drug. Quantitative cell uptake results for 24 hours. The intake rates of MCF-7 for CSO-SA/DOX and CSO-FBA-SA/ DOX nanoparticles were the same $(p>0.1)$ and free DOX $(p<0.05)$. This is caused by the strong affinity of the mesoporous multifunctional nanomaterial hydrophobic aliphatic chain membrane and the effective invasion of cells during the active drug transport through the nanoparticles.

In this study, an LC-MS/MS analysis method for the determination of TID-101 in rat plasma was established. After methodological investigation, this method is fast, sensitive, and specific and can meet the needs of pharmacokinetic research. The drug concentration at different times is shown in Table 2. TID-101 showed a good linear relationship between 100 and $3000 \mathrm{ml}(r 2=0.9996)$, the sample recovery rate was in the range of $83 \%-86 \%$ (all RSD $<15 \%$ ), and the matrix effect was in the range of $89.62 \%-91.92 \%$. Within $\mathrm{RSD}<15 \%$, it meets the requirements of biological samples. The intraday and interday precision (RSD) are less than $15 \%$, the stability of the sample during storage and analysis is good, and the relative error $\mathrm{RE}$ is reasonable. The $\mathrm{pH}$ responsiveness of mesoporous multifunctional nanomaterials is determined by the measurement of the change of the average hydrodynamic diameter at different $\mathrm{pH}$ values. The ATRP method is used to design functional polymer drug carriers and their controlled release behavior. When the $\mathrm{pH}$ is maintained at 3-4, the mesoporous multifunctional nanomaterials maintain a low $\mathrm{Dh}$; when the $\mathrm{pH}$ increases to 5-6, Dn shows a linear growth trend; when the $\mathrm{pH}$ is higher than 6 , Dh increases significantly. The reason is attributed to the $\mathrm{pH}$ responsiveness of the PAA block. This phenomenon not only reveals the $\mathrm{pH}$-responsive characteristics of mesoporous multifunctional nanomaterials, but also shows its potential as a smart drug carrier.

\subsection{Targeted Therapy Analysis of Mesoporous Multifunctional} Nanomaterials. The drug release image obtained after the mesoporous multifunctional nanomaterial-loaded drug released in PBS buffer at $\mathrm{pH}=7.5$ for 15 days is shown in Figure 2. It can be seen from the figure that the drug release process can be divided into two stages of rapid release and stable and slow release. The release amount of TNT samples in the rapid release stage was about $88.7 \%$, which lasted 5 hours, and the release amount of TNTs-P25 samples at this stage was about $79 \%$. The proportion of the drug release amount in the rapid release stage after modification of P25 decreased. At this stage, the drug IBU attached to the mesoporous multifunctional nanomaterial is released. After $5 \mathrm{~h}$, it is the slow release phase of the drug, which releases ibuprofen adsorbed inside and deep in the nanotubes and lasts longer. Comparing the release curve of the unmodified P25 sample, it can be seen that the P25 sample was modified by hydrothermal treatment. During the slow release stage, the amount of drug released increased significantly, which may be because the specific surface area and the roughness of the wall of the mesoporous multifunctional nanotube array after P25 modification were greatly increased, which was more conducive to the adsorption of drugs and the inside of the tube and the wall. The release of the drug has a delayed effect, and the total drug release has increased by $33 \%$, which further shows that the modified mesoporous multifunctional nanomaterials are more conducive to the adsorption of the drug, which is consistent with the results of the previous analysis.

The release behavior of CLB and DOX from the mesoporous multifunctional nanomicelle combined drug delivery system under different conditions is shown in Figure 3. When GSH is not added, both CLB and DOX are slowly released, and the cumulative release of CLB is less than $6 \%$ within 200 minutes, basically not released, and the release of DOX is relatively fast, nearly $18 \%$; when $3 \mathrm{SH}$ of GSH is added, the release rate increased slightly, reaching $9 \%$ and $23 \%$, respectively. When $10.0 \mathrm{~mol}$ MGSH was added, the release of DOX increased rapidly, reaching a maximum release rate of $86.5 \%$ at $50 \mathrm{~min}, \mathrm{CLB}$ was also released quickly at $30-90 \mathrm{~min}$, and the maximum cumulative release rate was $85.6 \%$. The results show that the constructed mesoporous multifunctional nanomicelle combined drug delivery method has obvious reduction sensitivity. Doxorubicin enters the core of the carrier through physical embedding and is first released relative to the CLB integrated into the 


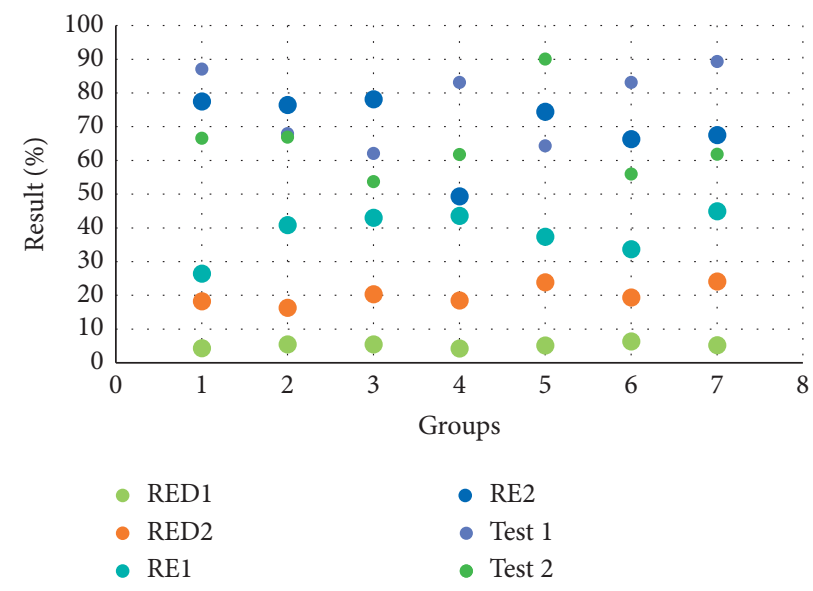

Figure 1: Muscle healing in different groups.

TABle 2: Drug concentration at different times.

\begin{tabular}{|c|c|c|c|c|c|c|c|}
\hline \multirow{2}{*}{ Test drug } & \multirow{2}{*}{ Concentration } & \multicolumn{3}{|l|}{ Daytime } & \multicolumn{3}{|l|}{ Within days } \\
\hline & & Determination of concentration & RED & $\mathrm{RE}$ & Determination of concentration & RSD & $\mathrm{RE}$ \\
\hline \multirow{3}{*}{ TID-101 } & 40 & $35 \pm 2$ & $6.2 \%$ & $-3.9 \%$ & $37 \pm 2$ & $5.8 \%$ & $-4.2 \%$ \\
\hline & 400 & $320 \pm 50$ & $2.6 \%$ & $-4.8 \%$ & $330 \pm 50$ & $2.3 \%$ & $-5.1 \%$ \\
\hline & 4000 & $3200 \pm 100$ & $2.7 \%$ & $-6.6 \%$ & $3200 \pm 200$ & $2.5 \%$ & $-8.0 \%$ \\
\hline
\end{tabular}

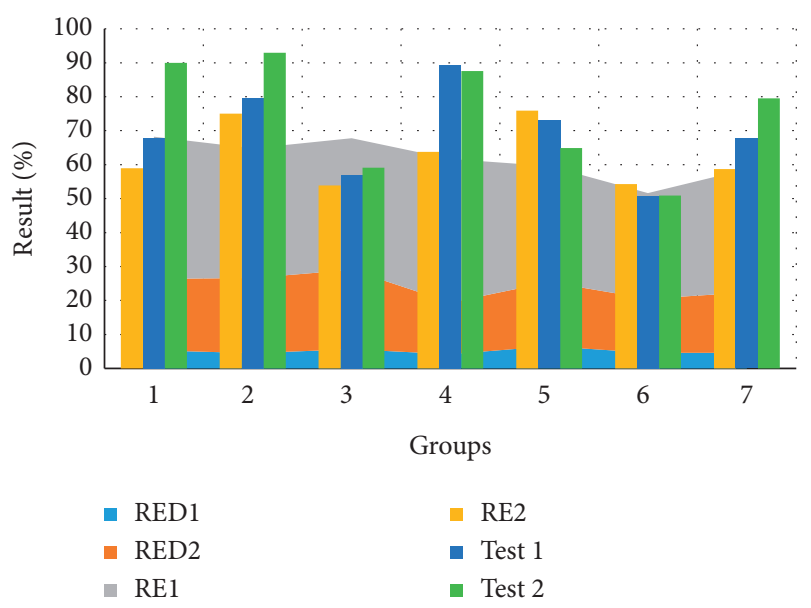

Figure 2: Drug release images obtained by mesoporous multifunctional nanomaterials loaded with drugs.

carrier through chemical bonds. At the same time, the release rate of the two under physiological conditions is very low, and the drug delivery system shows good stability. When reaching the injured muscle, both are released quickly under reducing stimulation. The mesoporous multifunctional nanomicelle lyophilized powder was configured into $1 \mathrm{mg} / \mathrm{mL}, 2 \mathrm{mg} / \mathrm{mL}$, and $5 \mathrm{mg} / \mathrm{mL}$ solutions with water, and all samples were placed in a constant temperature steam bath shaker $\left(25^{\circ} \mathrm{C}\right)$ and shaken at a constant speed for $10 \mathrm{~h}$ and then statically placed for $3 \mathrm{~h}$ (room temperature), and the particle size of the drug-loaded micelles was measured by a dynamic laser light scattering instrument. The average particle size was $11.2 \mathrm{~nm}, 15.4 \mathrm{~nm}$, and $13.5 \mathrm{~nm}$, respectively. The values of polydispersity index (PDI) are $0.4,0.3$, and 0.5 in order. Because TAT polypeptide can effectively bind to the nuclear transporter on the nuclear membrane, drugs mainly modify the TAT polypeptide on the surface of mesoporous silicon by covalent bond modification to achieve the nuclear fusion effect. The results show that the smaller the particle size of mesoporous silicon, the easier it is to enter the cell nucleus, and the confocal microscopy imaging effectively proves the targeting effect of mesoporous multifunctional nanomaterials on the cell nucleus.

4.3. Safety Analysis of Mesoporous Multifunctional Nanomaterials on Cells. The survival rate of cells under the environment of mesoporous multifunctional nanomaterials is shown in Figure 4. As a carrier material for drug delivery, mesoporous multifunctional nanomaterials have good biocompatibility and will not destroy the normal structure of cells. Cytotoxicity tests are commonly used to evaluate the safety of mesoporous multifunctional nanomaterial carriers on cells. Therefore, in the nanoparticle transport and absorption experiments, different concentrations of $100 \mu \mathrm{g} / \mathrm{ml}$ nanoparticles were used. The cell safety of mesoporous multifunctional nanomaterials and pSLN particles with different modification ratios was investigated by using the tetrazolium blue colorimetric method (MTT assay). MTT method is used for cytotoxicity detection. Exogenous pigments are produced in the mitochondria of living cells under the reduction of succinate dehydrogenase to produce waterinsoluble purple crystalline methylamine, which is positively correlated with the number of cells. Formazan can be 


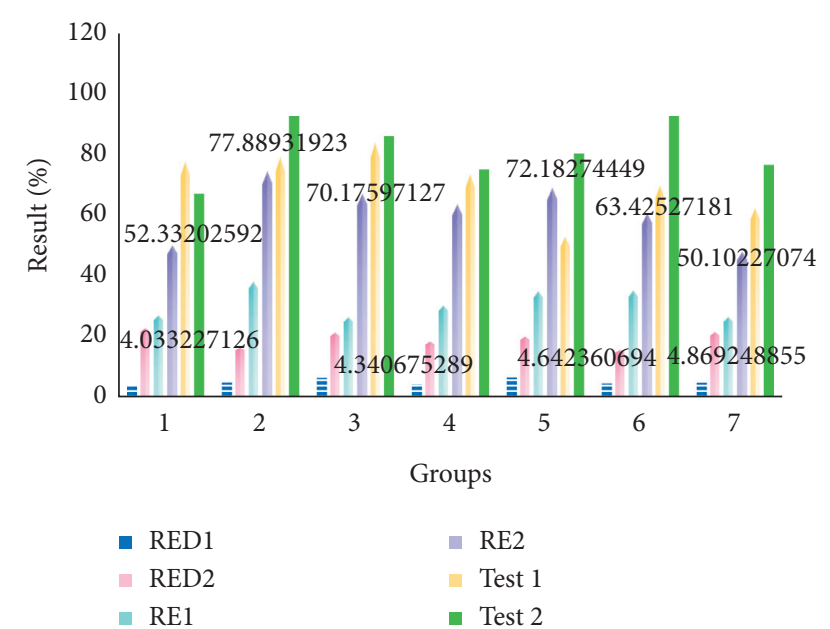

FIGURE 3: Simultaneous release behavior of CLB and DOX from the mesoporous multifunctional nanomicelle combined drug delivery system under different conditions.

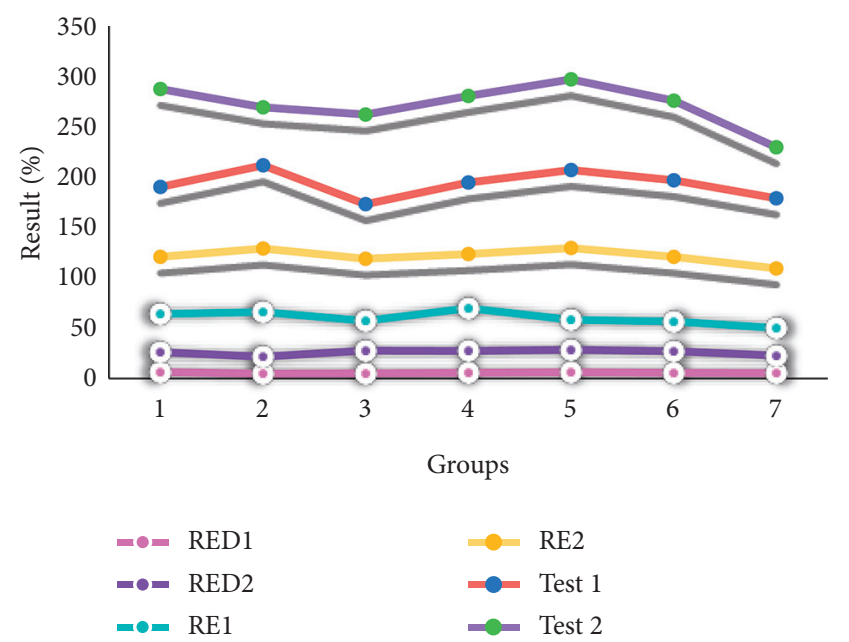

FIgURE 4: Cell survival rate in the environment of mesoporous multifunctional nanomaterials.

dissolved in DMSO, and the photometric value of cell transport studies of lipid-absorbing nanocarriers and PEGmodified lipid nanocarriers can be measured by enzymelinked immunoassay at a wavelength of $570 \mathrm{~nm}$, which can indirectly reflect cell growth and proliferation activity. By the MTT method, the half lethal amount of SLN and pSLN muscle cells can be measured about $300 \mu \mathrm{g} / \mathrm{ml}$. In addition, as the modification ratio of mesoporous multifunctional nanomaterials increases, the cytotoxicity of the carrier decreases. The results show that the mesoporous multifunctional nanomaterials have low cytotoxicity. When the nanoparticle concentration is $90 \mu \mathrm{g} / \mathrm{ml}$, the cell survival rate is close to $100 \%$, and the cell activity is not affected.

In addition, when the sustained drug release time exceeds $72 \mathrm{~h}$, some drugs are still released, which shows that the mesoporous multifunctional nanomaterials can significantly prolong the circulation time of the drug, reduce the probability of the drug being degraded in the body fluid circulation and reduce the toxicity caused by the high concentration of drug and side effects, and improve drug efficacy. Comparing the drug release of $\beta$-HPC/GFT at two $\mathrm{pH}$ values, the release rate is faster than the neutral $\mathrm{pH} 7.4$ under the condition of low $\mathrm{pH} 5.0$, which shows that the drug release depends on $\mathrm{pH}$, and this $\mathrm{pH}$-dependent release behavior is beneficial to drug release in the lysosome and muscle damaging environment. Therefore, we can imagine that the cationic polymer engulfed into the cell through the cell released the drug in the acidic lysosome of the cell, and due to the "proton sponge efficiency" of the mesoporous multifunctional nanomaterials, the lysosomal membrane was broken, and thus, the drug was released into the cytoplasm to play a role.

\subsection{Rehabilitation Intervention of Sports Training on Muscle} Injury. Sports training refers to various buffering actions that the human body gradually changes from a tense action state to a relatively quiet state. The quality of relaxation activities is directly related to the improvement of sports and fitness levels in the process of physical health. After exercise, relax activities, eliminate fatigue, adjust internal organs, and bring good effects to mental relaxation. Although relaxing and organizing activities have a very important role and significance in improving sports ability, in actual teaching and training, they often do not attract enough attention and are usually only optional. Psychological factors are an indirect factor to avoid or reduce sports injuries. If sports injuries occur during bodybuilding training, there must be corresponding psychological fear and psychological fatigue. If they cannot be eliminated in time, it will have a negative impact on the recovery of sports injuries. Therefore, the rational use of the organic combination of muscle relaxation and psychological relaxation can better promote the elimination of physical fatigue.

\section{Conclusions}

This study explored the drug loading function of nanocapsules. First, it shows high loading capacity and efficiency. Secondly, under different $\mathrm{pH}$ and reducing agent conditions, the release behavior shows a great difference, and the content of the drug also changes dynamically with $\mathrm{pH}$, proving the existence of $\mathrm{pH}$ and reduction reactivity.

At present, mesoporous multifunctional nanomaterials have many advantages, such as proper improvement of drug dynamics and biological distribution, improvement of biodiversity, and reduction of side effects. However, the method of further achieving the localization and release of drugs at muscle injury sites is still a compelling research topic. The specificity of local drug damage is high, and the dosage can be reduced to a certain extent, but it will be limited by the damage category. Systemic administration is still the preferred method of administration for most types of muscle damage. Therefore, targeted therapy at the site of muscle injury is the focus. 
The nanodrug carrier encapsulates the drug in a closed environment, which can improve the stability of the drug to the external environment, prevent the drug from decomposing before reaching the lesion, and reduce the systemic drug concentration. Controlled release can also maintain a stable drug concentration in the blood and reduce drug toxicity and side effects. Cytotoxicity indicates that it is very safe for cells. Mesoporous multifunctional nanomaterials will become the focus of medical development.

\section{Data Availability}

The data used to support the findings of the study are available from the corresponding author upon request.

\section{Conflicts of Interest}

The authors declare that they have no conflicts of interest.

\section{References}

[1] Z. Yanyan, W. Minghao, W. Mingjie et al., "Multifunctional carbon-based nanomaterials: applications in biomolecular imaging and therapy," Acs Omega, vol. 3, no. 8, pp. 9126-9145, 2018.

[2] R. Paul, F. Du, L. Dai et al., "3D heteroatom-doped carbon nanomaterials as multifunctional metal-free catalysts for integrated energy devices," Advanced Materials, vol. 31, no. 13, Article ID 1805598, 2019.

[3] L. Li, C. Chen, H. Liu et al., "Cancer therapy: multifunctional carbon-silica nanocapsules with gold core for synergistic photothermal and chemo-cancer therapy under the guidance of bimodal imaging (advanced functional materials 24/2016)," Advanced Functional Materials, vol. 26, no. 24, pp. 4252-4261, 2016.

[4] S. A. Santos, E. T. Silva, A. V. Caris et al., "Vitamin E supplementation inhibits muscle damage and inflammation after moderate exercise in hypoxia," Journal of Human Nutrition and Dietetics, vol. 29, no. 4, pp. 516-522, 2016.

[5] L. Zhang, T. Liu, and Y. Chen, "Magnetic conducting polymer/mesoporous $\mathrm{SiO} 2$ yolk/shell nanomaterials: multifunctional nanocarriers for controlled release of doxorubicin," Rsc Advances, vol. 6, no. 11, pp. 8572-8579, 2016.

[6] M. Marisa and S. Miriam, "Multifunctional nanomaterials: design, synthesis and application properties," Molecules, vol. 22, no. 2, p. 243, 2017.

[7] W. Gong, Z. Hu, and Y. Liang, "Graphene-based multifunctional nanomaterials in cancer detection and therapeutics," Journal of Nanoence \& Nanotechnology, vol. 18, no. 8, pp. 5155-5750, 2018.

[8] L. Qiu, Z. He, and D. Li, "Multifunctional cellular materials based on 2D nanomaterials: prospects and challenges," $A d$ vanced Materials, vol. 30, no. 4, Article ID 1704850, 2018.

[9] G. Yang, H. Gong, T. Liu et al., "Mesoporous silica-coated WS2@ $\mathrm{Fe}_{3} \mathrm{O}_{4}$ nanosheets as a multifunctional theranostic nanocarrier for combination therapy of cancer," Nanomedicine Nanotechnology Biology \& Medicine, vol. 12, no. 2, p. 523, 2016.

[10] Z. Libo, J. Ying, L. Yubin et al., "Mesoporous carbon nanospheres as a multifunctional carrier for cancer theranostics," Theranostics, vol. 8, no. 3, pp. 663-675, 2018.

[11] D. Wang, N. B. Saleh, W. Sun et al., "Next-generation multifunctional carbon-metal nanohybrids for energy and environmental applications," Environmental Ence and Technology, vol. 53, no. 13, pp. 7265-7287, 2019.

[12] A. A. Oun, S. Shankar, and J. W. Rhim, "Multifunctional nanocellulose/metal and metal oxide nanoparticle hybrid nanomaterials," C R C Critical Reviews in Food Technology, vol. 60, no. 3, pp. 435-460, 2020.

[13] T. Wiewelhove, J. Fernandez-Fernandez, C. Raeder et al., "Acute responses and muscle damage in different high-intensity interval running protocols," The Journal of Sports Medicine and Physical Fitness, vol. 56, no. 5, pp. 606-615, 2016.

[14] C. K. Deli, I. G. Fatouros, V. Paschalis et al., "A comparison of exercise-induced muscle damage following maximal eccentric contractions in men and boys." Pediatric Exercise Science, vol. 29, no. 3, p. 316, 2017.

[15] O. Eisuke and T. Yosuke, "Eicosahexanoic acid (EPA) and docosahexanoic acid (DHA) in muscle damage and function," Nutrients, vol. 10, no. 5, p. 552, 2018.

[16] De Anta-Díaz, Belén, J. Serralta-Gomis, A. Lizaur-Utrilla et al., "No differences between direct anterior and lateral approach for primary total hip arthroplasty related to muscle damage or functional outcome," International Orthopaedics, vol. 40, no. 10, pp. 2025-2030, 2016.

[17] n P. AbiÃ, J. Del Coso, and J. J. Salinero, "Muscle damage produced during a simulated badminton match in competitive male players," Research in Sports Medicine, vol. 24, no. 1, pp. 104-117, 2016.

[18] J. Del Coso, M. Valero, J. José Salinero et al., "ACTN3 genotype influences exercise-induced muscle damage during a marathon competition," European Journal of Applied Physiology, vol. 117, no. 3, pp. 409-416, 2017.

[19] M. A. Brown, E. J. Stevenson, and G. Howatson, "Whey protein hydrolysate supplementation accelerates recovery from exercise-induced muscle damage in females," Applied Physiology, Nutrition, and Metabolism, vol. 43, no. 4, p. 324, 2018.

[20] F. Damas, K. Nosaka, C. Libardi et al., "Susceptibility to exercise-induced muscle damage: a cluster Analysis with a large sample," International Journal of Sports Medicine, vol. 37, no. 08 , pp. 633-640, 2016.

[21] E. D. Withee, K. M. Tippens, R. Dehen et al., "Effects of methylsulfonylmethane (MSM) on exercise-induced oxidative stress, muscle damage, and pain following a half-marathon: a double-blind, randomized, placebo-controlled trial," Journal of the International Society of Sports Nutrition, vol. 14, no. 1, p. 24, 2017.

[22] M. Malakoutian, J. Street, H. J. Wilke et al., "Role of muscle damage on loading at the level adjacent to a lumbar spine fusion: a biomechanical analysis," European Spine Journal, vol. 25, no. 9, pp. 2929-2937, 2016. 